\title{
Raltegravir, etravirine and darunavir-ritonavir: a safe and successful rescue regimen in highly treatment-experienced HIVI-infected patients
}

\author{
A Imaz*1, S Villar del Saz¹, MA Rivas², A Curran11, E Caballero1, V Falco ${ }^{1}$, \\ M Crespo${ }^{1}$, I Ocana ${ }^{1}, M$ Diaz$^{1}$, E Ruiz de Gopegui ${ }^{2}$, M Riera $^{2}$ and E Ribera ${ }^{1}$
}

Address: ${ }^{1}$ Hospital Vall d'Hebron, Barcelona, Spain and ${ }^{2}$ Son Dureta, Palma de Mallorca, Spain

* Corresponding author

from Ninth International Congress on Drug Therapy in HIV Infection

Glasgow, UK. 9-13 November 2008

Published: 10 November 2008

Journal of the International AIDS Society 2008, I I (SuppI I):P40 doi:I0.I I86/I758-2652-I I-SI-P40

This abstract is available from: http://www.jiasociety.org/content/II/SI/P40

(C) 2008 Imaz et al; licensee BioMed Central Ltd.

\section{Background}

Ritonavir-boosted darunavir (DRV/r) and etravirine (ETR) in DUET trials and raltegravir (RAL) in BENCHMRK trials have shown high rates of virological responses in treatment-experienced patients with multi-drug resistant HIVinfection, particularly when used in association with two more fully active antiretroviral drugs. However, the combination of DRV/r, ETR and RAL has not been evaluated.

\section{Methods}

Prospective observational study of all consecutive HIV-1 infected adults with virological failure and very limited treatment options, who started a new salvage regimen with DRV/r (600/100 mg twice daily), ETR (200 mg twice daily) and RAL (400 mg twice daily), from April 2007 through January 2008 in two hospitals in Spain. Clinical evaluation and immunological, virological and biochemical tests were performed at baseline and at week 4, 12 and 24.

\section{Summary of Results}

Overall there were 32 patients: 23 were male and all Caucasian. Median baseline characteristics were: age 44 years, 16 years since HIV diagnosis, 13 years on antiretroviral therapy (ART), nine prior HAART regimens, CD4 count 261 cells/mL and HIV-1 RNA $4.2 \log 10$ copies/mL. Sixteen $(50 \%)$ and 14 (44\%) were enfuvirtide and tipranavir experienced, respectively. At baseline, all patients harboured three-class resistance mutations, with a median of five IAS-USA primary PI resistance mutations (75\% had four or more), two NNRTI mutations and five NRTI mutations (four TAMs) at baseline. Three patients (9\%) harboured three ETR resistance mutations. All patients were DRV-naïve and had a median of one (0-3) DRV resistance mutation. The percentage of patients $($ ITTnc $=f)$ with HIV1-RNA below 50 copies/mL was $63 \%$ (20/32) at week $4,81 \%(26 / 32)$ at week 12 and 94\% (30/32) at week 24 . Only two patients did not achieved virological suppression: one stopped treatment at week 4 and the other had poor adherence overtime. Median CD4 cell count increased 30, 73 and 103 cells/mL at week 4, 12 and 24, respectively. None of the patients had adverse events leading to discontinuation of the regimen.

\section{Conclusion}

The combination of DRV/r, ETR and RAL was a highly effective and well tolerated antiretroviral salvage regimen in patients with multi-drug resistant HIV1-infection. 